\title{
Presentation in Dirihe- Neumann Plans and Compiling of Progam with Programmming Language $\mathrm{C}+$
}

\author{
Prof. Ass. Dr. Naim Baftiu \\ University of Prizren "Ukshın Hoti”, Faculty of Computer Sciences, Republic of Kosovo \\ Dr. Veli Lecaj \\ University of Prizren "Ukshın Hoti”, Faculty of Economy, Republic of Kosovo \\ Dr. Sc. Eflorim Hajra \\ Institute of Scientific Researches "INKOS", Department of Geology Republic of Kosovo
}

\section{Doi:10.5901/ajis.2015.v4n2p539}

\section{Abstract}

\begin{abstract}
The problem with supply of industrial water nowadays is becoming very serious for the reason that the industry requires more quantity of clear water. Since natural water from the rivers and other sources doesn't fulfil the necessities, it is before using it in industry in many cases is prepared and processed by means of filtration. One of the most useful way of water filtration is programming in programming language $\mathrm{C}+$. This program has the objective of calculation of water filtration in special cases in order to achieve the creation of shorter time and more accurate way. The goal of this work is calculation of water filtration and studying of a water zone in village Sibovc, Kosovo of $1 \mathrm{~km}$ area with the method of Fundamental Elements and statistic processing with DIRHILE-NEUMANN Method which is inserted in Programming language C+. This programming language has found wide applications not only in the field of water but also those with thermo hydro-dynamic character, calculation of water filtration, prescription of physical-chemical particularities etc. With this advanced program C+ is used a given model with number of nodes 10 and number of elements 10, whereas limited conditions with DIRIHLE-NEUMANN Method in e definite level are in the contour 3-7 charge will be $\Phi_{3}=\Phi_{7=100} \mathrm{~m}$, whereas in contour 8 the charge will be $\Phi_{8}=200 \mathrm{~m}$, and in contour 10 the charge will be $\Phi 10=300 \mathrm{~m}$, flux of the source is : $q 1=q 5=q 9=2, q 2=2.5 \mathrm{m3} /$ day, coefficient of filtration $k=100$ $m /$ day.
\end{abstract}

Keywords: water filtration, Dirihle-Neumann Method, programming language C+, contours and Filtration coefficient.

\section{Economic Aspect and the Importance of Water in Society}

Hygienically clean water for drinking is a prerequisite for good health of the population, in connection with which the (WHO, 2010: 35) World Health Organization water quality and supply has ranked twelve basic indicators of health status the population of a country. So it turns out that water is vital for public health, economic development, and national security, the establishment of social welfare and beyond.

Water is a limited resource, thus, provides clean water and safe for current needs and for future generations represents one of the main challenges of Kosovar state. Especially challenging is presented providing water to all citizens of Kosovo, and Kosovo agriculture businesses for a sustainable period of time, considering the great climatic changes the world, whose split Kosovo cannot be my municipality. This requires a multidimensional scientific study regarding the research, extraction, filtration and water usage sure to rinse having constant access and unlimited water supply, this issue that affects the economic development of the public health and welfare of citizens Kosovo.

Scientists explain that our planet consists of $75 \%$ water, but only $1 \%$ of water is available for drinking. Time needed to watch the waters in the territory of Kosovo from another point of view, based on the fact that during the movement of water in nature, polluted air and from the ground, often by human negligence by large quantities of water black and other water discharged into rivers and lakes, including factories, utilities, homes, other not forgetting or agricultural pollution from the use of nitrogen fertilizers, pesticides, herbicides other.

On the other hand the growth of population and its movements, change of land use, including the characteristics of the weather and climatic conditions pose the necessity of research and efficient management of water use. The 
importance that separate the one hand is the increased use of water demand for energy production and agriculture sector after Kosovo's population continues to grow constantly, what the resources and the availability of water is not always coincide with the location of the population, on the other hand.

In this case it requires deep drilling research and water resources and the necessary transfer of resources to the areas that need it as is the case with the municipality of Orahovac. In this municipality are two drilling made from 100 meters depth, it is found water, and are doing all the work associated with the completion of this project by providing water flow of 3.3 litters per second to as the project has cost 29.600 euros. This project has provided sufficient supply of both villages with drinking water and is believed to be a sustainable supply that would justify the cost. Therefore we end the economic rationale logically justified and accepted through the slogan: "Access to water and sanitation - inalienable human right"

\section{Introduction}

Water filtration is a basic operation whose purpose is that its current amount to define in correctly way to requested location. The whole Sibovc's area has a surface about $16 \mathrm{~km}$ width a maximum width of $3.8 \mathrm{~km}$ and length of about $6 \mathrm{~km}$. Surface water that way affect is mainly in small amount and have no permanent leak. This area is characterized by a continental climate with dry and hot summer and indifferent winter temperatures depending on the influence areas of high pressure from Siberia or low pressure areas of the Atlantic Ocean.

The average annual temperature is around $+10 \mathrm{C}$. The lowest temperature measured is $25.2 \mathrm{C}$. Rainfall data were collected from different sources. In 2000 Kosovo Hydrometeorology Institute conducted a study where is submitted monthly average for the period of 25 years. The database is completed with exiting evaluation for the period of 2004 to 2013. Calculated average annual rainfall of $600 \mathrm{~mm}$. Minimal rainfall data are described in 1990 with $372 \mathrm{~mm}$, maximum annual rainfall are recorded in 1995 with $1010 \mathrm{~mm}$. This area forms a slight plain which is limited by hills and mountains and it contains a well-developed hydrological network with a leading collector which is Sitnica River. This river runs through the base from north to south and drainage about $80 \%$ of surface accumulated water in the northern direction. Other rivers in the vicinity are Drenica River in the west and Llapi River in the east. The flow of Sitnica River varies between the minimum of $0.5-1.5 \mathrm{~m} 3 / \mathrm{s}$ and the maximum of $50-120 \mathrm{~m} 3 / \mathrm{s}$ with an average of $5-10 \mathrm{~m} 3 / \mathrm{s}$. In the periods of flooding, the low of river reaches the width to $1000 \mathrm{~m}$ in the flooded areas.

Table 1/1.The comparison of water qualities.

\begin{tabular}{|c|c|c|c|c|}
\hline & & Minimum & Average & Maximum \\
\hline Units & & Analyzed area Sibovci & Analyzed area Sibovci & Analyzed are Sibovci \\
\hline Value of $\mathrm{pH}$ & & 6.7 & 7.8 & 8.7 \\
\hline The electrical conductors & $\mu S / \mathrm{cm}$ & 175.0 & $1,381.0$ & $3,700.0$ \\
\hline Chloride & $\mathrm{mg} / \mathrm{l}$ & 4.5 & 90.0 & 290.0 \\
\hline Sulphate & $\mathrm{mg} / \mathrm{l}$ & 75.0 & 924.0 & $1,741.0$ \\
\hline Hidrokarbonati & $\mathrm{mg} / \mathrm{l}$ & 232.0 & 447.0 & 600.0 \\
\hline Nitrate & $\mathrm{mg} / \mathrm{l}$ & 0.0 & 10.3 & 72.0 \\
\hline Consumption of $\mathrm{KMnO}_{4}$ & $\mathrm{mg} / \mathrm{l}$ & 3.0 & 45.0 & 183.0 \\
\hline
\end{tabular}

Exploitation of underground water concentrates on private wells that are due to a depth of 10 to $15 \mathrm{~m}$ within underground surface. Produced quantities are given by the Institute in (1985) with $Q=3(1 / \mathrm{min})$ to $Q=11(1 / \mathrm{min})$ with a maximum from $\mathrm{Q}=54(1 / \mathrm{min})$ which can be judged as hydraulic conductivity rate from $\mathrm{k}_{\mathrm{f}}=10^{-9} \mathrm{~m} / \mathrm{s}$ to $\mathrm{k}_{\mathrm{f}}=10^{-6} \mathrm{~m} / \mathrm{s}$. 


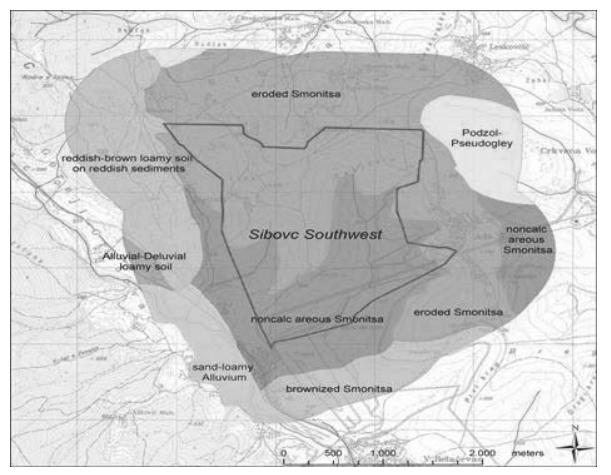

Figure 1/1. Location of the extent of area of water filtration, which is calculated with finite elements in the plan of DIRIHLE-NEUMANN by C + program.

\section{The Method of Difference and Finite Elements of Water Filtration - The Plan Problem Dirihle-Neumann}

Finite elements method applies not only to things in the pattern of elastic fields but also to those of hydrodynamic thermal character, filtering etc. It was taken a plan area as in diagram 1.1 and is presented points of contours-nodes.

The coordinates of contour-nodes in relation to the chosen coordinate system are shown in the table.

Table: The coordinates of nodes in the defined area

\begin{tabular}{|c||c|c|c|c|c|c|c|c|c|c|}
\hline Nr. of nodes & 1 & 2 & 3 & 4 & 5 & 6 & 7 & 8 & 9 & 10 \\
\hline \hline Abscissa X & 1 & 1 & 1 & 2 & 2 & 3 & 3 & 4 & 4 & 5 \\
\hline \hline Ordinate Y & 3 & 5 & 7 & 5 & 1 & 3 & 7 & 5 & 1 & 3 \\
\hline
\end{tabular}

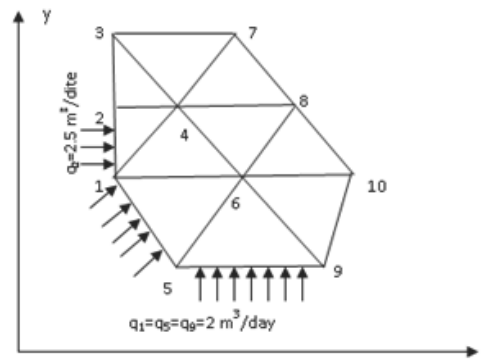

The Diagram: 1/1 Definition-outlines of the study area-filtration

Dirihle boundary conditions with (a certain level) are:

In the contour 3-7 loads will be: $\Phi_{3}=\Phi_{7}=100 \mathrm{~m}$ and the contour 8 loads will be $\Phi_{8}=200 \mathrm{~m}$, while the contour 10 loads will be $\Phi_{10}=300$.

The flux of resources is: $q_{1}=q_{5}=q_{9}=2, q_{2}=2.5 \mathrm{~m}^{3} /$ day

Coefficient of Filtration: $k=100 \mathrm{~m} /$ day

$\Phi=\Phi_{-}^{+} c o n s \tan t$ Does not change the choice of the differential equation

$\frac{\partial}{\partial x}\left(\frac{k x}{\mu} \frac{\partial \bar{\Phi}}{\partial x}+\frac{\partial}{\partial y}\left(\frac{k y}{\mu} \frac{\partial \bar{\Phi}}{\partial y}\right)+Q=0\right.$

Which means it can be used either load conditions even zero. According to the finite elements method given area divide into basic geometric finite area for example to our case triangular. 
Solution: In the given model:

The number of nodes is 10

The number elements is 10

The topless of elements are:

$$
\begin{aligned}
& \Delta_{7}=\Delta_{8}=1 \\
& \Delta_{1}=\Delta_{2}=\Delta_{3}=\Delta_{4}=\Delta_{5}=\Delta_{6}=\Delta_{9}=\Delta_{10}=2
\end{aligned}
$$

Find the system of equations for each element considering the matrix formula:

$$
\begin{aligned}
& \frac{k}{4 \Delta}\left[\begin{array}{ccc}
b_{1}^{2} & b_{1} b_{2} & b_{1} b_{3} \\
b_{2} b_{1} & b_{2}^{2} & b_{2} b_{3} \\
b_{3} b_{1} & b_{3} b_{2} & b_{3}^{2}
\end{array}\right]+\left[\begin{array}{ccc}
c_{1}^{2} & c_{1} c_{2} & c_{1} c_{3} \\
c_{2} c_{1} & c_{2}^{2} & c_{2} c_{3} \\
c_{3} c_{1} & c_{3} c_{2} & c_{3}^{2}
\end{array}\right] x\left\{\begin{array}{l}
\Phi_{1} \\
\Phi_{2} \\
\Phi_{3}
\end{array}\right\}+\frac{Q \Delta}{3}\left\{\begin{array}{l}
l \\
l \\
l
\end{array}\right\}+\frac{q L}{2}\left\{\begin{array}{l}
l \\
l \\
0
\end{array}\right\}=\left\{\begin{array}{l}
0 \\
0 \\
0
\end{array}\right\} \\
& a_{1}=x_{2} y_{3}-x_{3} y_{2} b_{1}=y_{2}-y_{3} \quad c_{1}=x_{3}-x_{2} \\
& a_{2}=x_{3} y_{1}-x_{1} y_{3} \quad b_{2}=y_{3}-y_{1} \quad c_{2}=x_{1}-x_{3} \\
& a_{3}=x_{1} y_{2}-x_{2} y_{1} \quad b_{3}=y_{3}-y_{3} \quad c_{3}=x_{2}-x_{1}
\end{aligned}
$$

For the number of nodes is chosen one direction:

The first element:

The coordinates:

\begin{tabular}{|c|c|c|}
\hline Nodes & $\mathrm{X}$ & $\mathrm{Y}$ \\
\hline 1 & 1 & 3 \\
\hline 5 & 2 & 1 \\
\hline 6 & 3 & 3 \\
\hline
\end{tabular}

$$
\begin{array}{llll} 
& a_{1}=x_{2} y_{3}-x_{3} y_{2} \Rightarrow a_{1}=3 & b_{1}=y_{2}-y_{3} \Rightarrow b_{1}=-2 & c_{1}=x_{3}-x_{2} \Rightarrow c_{1}=3 \\
& a_{2}=x_{3} y_{1}-x_{1} y_{3} \Rightarrow a_{2}=6 & b_{2}=y_{3}-y_{1} \Rightarrow b_{2}=0 & c_{2}=x_{1}-x_{3} \Rightarrow c_{2}=6 \\
\text { Deter =4 } & a_{3}=x_{1} y_{2}-x_{2} y_{1} \Rightarrow a_{3}=-5 & b_{3}=y_{3}-y_{3} \Rightarrow b=2 & c_{3}=x_{2}-x_{1} \Rightarrow b=-5
\end{array}
$$

After replacement the elementary matrix will be:

$$
\left[\begin{array}{ccc}
62.5 & -25 & -37.5 \\
-25 & 50 & -25 \\
-37.5 & -25 & 62.5
\end{array}\right]
$$

The second element:

The coordinates:

\begin{tabular}{|c|c|c|}
\hline Nodes & $\mathrm{X}$ & $\mathrm{Y}$ \\
\hline 5 & 2 & 1 \\
\hline 9 & 4 & 1 \\
\hline 6 & 3 & 3 \\
\hline
\end{tabular}

$$
\begin{array}{lll}
a_{1}=9 & b_{1}=-2 & c_{1}=9 \\
a_{2}=-3 & b_{2}=2 & c_{2}=-3
\end{array}
$$

Deter $=4 \quad a_{3}=-2 \quad b_{3}=0 \quad c_{3}=-2$

After replacement the elementary matrix will be:

$$
\left[\begin{array}{ccc}
62.5 & -37.5 & -25 \\
-37.5 & 62.5 & -25 \\
-25 & -25 & 50
\end{array}\right]
$$

The third element: The coordinates:

\begin{tabular}{|c|c|c|}
\hline Nodes & $X$ & $Y$ \\
\hline 6 & 3 & 3 \\
\hline 9 & 4 & 1 \\
\hline 10 & 5 & 3 \\
\hline
\end{tabular}




$$
\begin{aligned}
& a_{1}=7 \quad b_{1}=2 \quad c_{1}=7 \\
& a_{2}=6 \quad b_{2}=0 \quad c_{2}=6 \\
& \text { Deter }=4 \quad a_{3}=9 \quad b_{3}=2 \quad c_{3}=9
\end{aligned}
$$

After replacement the elementary matrix will be:

$$
\left[\begin{array}{ccc}
62.5 & -25 & -37.5 \\
-25 & 50 & -25 \\
-37.5 & -25 & 62.5
\end{array}\right]
$$

The fourth element:

The coordinates:

\begin{tabular}{|c|c|c|}
\hline Nodes & $\mathrm{X}$ & $\mathrm{Y}$ \\
\hline 2 & 1 & 5 \\
\hline 1 & 1 & 3 \\
\hline 4 & 2 & 5 \\
\hline
\end{tabular}

$$
\begin{array}{lll}
a_{1}=-1 & b_{1}=2 & c_{1}=-1 \\
a_{2}=5 & b_{2}=0 & c_{2}=5
\end{array}
$$

Deter $=2 \quad a_{3}=-2 \quad b_{3}=2 \quad c_{3}=-2$

After replacement the elementary matrix will be:

$$
\left[\begin{array}{ccc}
124 & -25 & -100 \\
-25 & 25 & 0 \\
100 & 0 & 100
\end{array}\right]
$$

The fifth element:

The coordinates:

\begin{tabular}{|c|c|c|}
\hline Nodes & $\mathrm{X}$ & $\mathrm{Y}$ \\
\hline 1 & 1 & 3 \\
\hline 6 & 3 & 3 \\
\hline 4 & 2 & 5 \\
\hline
\end{tabular}

$$
\begin{array}{lll}
a_{1}=9 & b_{1}=2 & c_{1}=9 \\
a_{2}=1 & b_{2}=2 & c_{2}=1
\end{array}
$$

Deter $=4 \quad a_{3}=-6 \quad b_{3}=0 \quad c_{3}=-6$

After replacement the elementary matrix will be:

$$
\left[\begin{array}{ccc}
62.5 & -37.5 & -25 \\
-37.5 & 62.5 & -25 \\
-25 & -25 & 50
\end{array}\right]
$$

The sixth element:

The coordinates:

\begin{tabular}{|c|c|c|}
\hline Nodes & $X$ & $Y$ \\
\hline 4 & 2 & 5 \\
\hline 6 & 3 & 3 \\
\hline 8 & 4 & 5 \\
\hline
\end{tabular}

$$
\begin{array}{lll}
a_{1}=3 & b_{1}=2 & c_{1}=3 \\
a_{2}=10 & b_{2}=0 & c_{2}=10
\end{array}
$$

Deter $=4 \begin{array}{llll}a_{3}=-9 & b_{3}=2 & c_{3}=9\end{array}$

After replacement the elementary matrix will be: 
$\left[\begin{array}{ccc}62.5 & -25 & -37.5 \\ -25 & 50 & -25 \\ -37.5 & -25 & 62.5\end{array}\right]$

The seventh element:

The coordinates:

\begin{tabular}{|c|c|c|}
\hline Nodes & $\mathrm{X}$ & $\mathrm{Y}$ \\
\hline 6 & 3 & 3 \\
\hline 10 & 5 & 3 \\
\hline 8 & 4 & 5 \\
\hline
\end{tabular}

$$
\begin{array}{rlll}
a_{1}=13 & b_{1}=-2 & c_{1}=13 \\
a_{2}=-3 & b_{2}=2 & c_{2}=-3 \\
\text { Deter }=4 & a_{3}=-6 & b_{3}=0 & c_{3}=-6
\end{array}
$$

After replacement the elementary matrix will be:

$\left[\begin{array}{ccc}62.5 & -37.5 & -25 \\ -37.5 & 62.5 & -25 \\ -25 & -25 & 50\end{array}\right]$

The eighth element:

The coordinates:

\begin{tabular}{|c|c|c|}
\hline Nodes & $\mathrm{X}$ & $\mathrm{Y}$ \\
\hline 6 & 3 & 3 \\
\hline 10 & 5 & 3 \\
\hline 8 & 4 & 5 \\
\hline
\end{tabular}

$$
\begin{array}{lll}
a_{1}=-5 & b_{1}=0 & c_{1}=-5 \\
a_{2}=9 & b_{2}=-2 & c_{2}=9
\end{array}
$$

Deter $=2 \quad a_{3}=-2 \quad b_{3}=2 \quad c_{3}=2$

After replacement the elementary matrix will be:

$$
\left[\begin{array}{ccc}
25 & -25 & 0 \\
-25 & 125 & -100 \\
0 & -100 & 100
\end{array}\right]
$$

The ninths element:

The coordinates:

\begin{tabular}{|c|c|c|}
\hline Nodes & $X$ & $Y$ \\
\hline 4 & 2 & 5 \\
\hline 7 & 3 & 7 \\
\hline 3 & 1 & 7 \\
\hline
\end{tabular}

$$
\begin{array}{lll}
a_{1}=14 & b_{1}=0 & c_{1}=14 \\
a_{2}=-9 & b_{2}=2 & c_{2}=-9
\end{array}
$$

Deter $=4 \quad a_{3}=-1 \quad b_{3}=-2 \quad c_{3}=-1$

After replacement the elementary matrix will be

$$
\left[\begin{array}{ccc}
50 & -25 & -25 \\
-25 & 62 & -37.5 \\
-25 & -37.5 & 62.5
\end{array}\right]
$$

The tenth element:

The coordinates: 


\begin{tabular}{|c|c|c|}
\hline Nodes & $\mathrm{X}$ & $\mathrm{Y}$ \\
\hline 4 & 2 & 5 \\
\hline 8 & 4 & 5 \\
\hline 7 & 3 & 7 \\
\hline
\end{tabular}

$$
\begin{array}{llll}
a_{1}=13 & b_{1}=-2 & c_{1}=13 \\
a_{2}=1 & b_{2}=2 & c_{2}=1 \\
\text { Deter }=4 & a_{3}=-10 & b_{3}=0 & c_{3}=-10
\end{array}
$$

After replacement the elementary matrix will be:

\begin{tabular}{|c|c|c|c|c|c|c|c|c|c|c|c|}
\hline Nodes & Loads & $\Phi_{1 \mathrm{~m}}$ & $\Phi_{2} \mathrm{~m}$ & $\Phi_{3} \mathrm{~m}$ & $\Phi_{4} \mathrm{~m}$ & $\Phi_{5} \mathrm{~m}$ & $\Phi_{6} \mathrm{~m}$ & $\Phi_{7} \mathrm{~m}$ & $\Phi_{8} \mathrm{~m}$ & $\Phi_{9 \mathrm{~m}}$ & $\Phi_{10 \mathrm{~m}}$ \\
\hline & & 62.5 & & & & -25 & -37.5 & & & & \\
\hline & & 62.5 & & & -25 & & -37.5 & & & & \\
\hline \multirow{4}{*}{1} & SIIM & 150 & 25 & 0 & 25 & 25 & 75 & 0 & 0 & 0 & 0 \\
\hline & Sorv & & 20 & 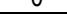 & 20 & $2 v$ & -10 & 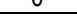 & 0 & 0 & 0 \\
\hline & & -25 & 125 & & -100 & & & & & & \\
\hline & & & 125 & -25 & -100 & & & & & & \\
\hline \multirow[t]{3}{*}{2} & SUM & -25 & 250 & -25 & -200 & 0 & 0 & 0 & 0 & 0 & 0 \\
\hline & & & -25 & 25 & 0 & & & & & & \\
\hline & & & & 62.5 & -25 & & & -37.5 & & & \\
\hline \multirow[t]{7}{*}{3} & SUM & 0 & -25 & 87.5 & -25 & 0 & 0 & -37.5 & 0 & 0 & 0 \\
\hline & & 0 & -100 & & 100 & & & & & & \\
\hline & & 0 & -100 & & 100 & & & & & & \\
\hline & & & & -25 & 50 & & 0 & -25 & & & \\
\hline & & & & & 62.5 & & & -25 & -37.5 & & \\
\hline & & & & & 62.5 & & -25 & & -37.5 & & \\
\hline & & -25 & & & 50 & & -25 & & & & \\
\hline \multirow[t]{3}{*}{4} & SUM & -25 & -200 & -25 & 425 & 0 & -50 & -50 & -75 & 0 & \\
\hline & & -25 & & & & 50 & -25 & & & & \\
\hline & & & & & & 62.5 & -25 & & & -37.5 & \\
\hline \multirow[t]{6}{*}{5} & SUM & -25 & 0 & 0 & 0 & 112.5 & -50 & 0 & 0 & -37.5 & 0 \\
\hline & & -37.5 & & & -25 & & 62.5 & & & & \\
\hline & & & & & & -25 & 50 & & & -25 & \\
\hline & & & & & & & 62.5 & & & -25 & -37.5 \\
\hline & & & & & -25 & & 50 & -25 & & & \\
\hline & & & & & & & -62.5 & -25 & & & -37.5 \\
\hline \multirow[t]{3}{*}{6} & SUM & -75 & 0 & 0 & -50 & -50 & 350 & 0 & -50 & -50 & -75 \\
\hline & & & & & -25 & & & 50 & -25 & & \\
\hline & & & & -37.5 & -25 & & & 62 & & & \\
\hline \multirow[t]{4}{*}{7} & SUM & 0 & 0 & -37.5 & -50 & 0 & 0 & 112.5 & -25 & 0 & 0 \\
\hline & & & & & -37.5 & & & -25 & 62.5 & & \\
\hline & & & & & & & -25 & & 50 & 0 & -25 \\
\hline & & & & & -37.5 & & -25 & & 62.5 & & \\
\hline \multirow[t]{3}{*}{8} & SUM & 0 & 0 & 0 & -75 & 0 & -50 & -25 & 175 & 0 & -25 \\
\hline & & & & & & & -25 & & & 50 & -25 \\
\hline & & & & & & -37.5 & -25 & & & 62.5 & \\
\hline \multirow[t]{3}{*}{9} & SUM & 0 & 0 & 0 & 0 & -37.5 & -50 & 0 & 0 & 112.5 & -25 \\
\hline & & & & & & & -37.5 & & -25 & & 50 \\
\hline & & & & & & & -37.5 & & & -25 & 62.5 \\
\hline 10 & SUM & 0 & 0 & 0 & 0 & 0 & -75 & -25 & -25 & -25 & 125 \\
\hline
\end{tabular}

$\left[\begin{array}{ccc}62.5 & -37.5 & -25 \\ -37.5 & 62.5 & -25 \\ -25 & -25 & 50\end{array}\right]$

After the replacement of elementary matrix we gain the table: $1 / 1$ 
By the summarized table we gain the system matrix.

$\left[\begin{array}{cccccccccc}150 & -25 & 0 & -25 & -25 & -75 & 0 & 0 & 0 & 0 \\ -25 & 250 & 0 & -200 & 0 & 0 & 0 & 0 & 0 & 0 \\ 0 & -25 & 87.5 & -25 & 0 & 0 & -37 & 0 & 0 & 0 \\ -25 & -200 & -25 & 425 & 0 & -50 & -50 & -75 & 0 & 0 \\ -25 & 0 & 0 & 0 & 112.5 & -50 & 0 & 0 & -37.5 & 0 \\ -75 & 0 & 0 & -50 & -50 & 350 & 0 & -50 & -50 & -75 \\ 0 & 0 & -37.5 & -50 & 0 & 0 & 112.5 & -25 & 0 & 0 \\ 0 & 0 & 0 & -75 & 0 & -50 & -25 & 175 & 0 & -25 \\ 0 & 0 & 0 & 0 & -37.5 & -50 & 0 & 0 & 112.5 & -25 \\ 0 & 0 & 0 & 0 & 0 & -75 & 0 & -25 & -25 & 125\end{array}\right]$

Whereas the matrix of the transformed system will be:

$\left[\begin{array}{cccccccccc}150 & -250 & 0 & -25 & -25 & -75 & 0 & 0 & 0 & 0 \\ -25 & 250 & 0 & -200 & 0 & 0 & 0 & 0 & 0 & 0 \\ 0 & 0 & 1 & 0 & 0 & 0 & 0 & 0 & 0 & 0 \\ -25 & -200 & 0 & 425 & 0 & -50 & 0 & 0 & 0 & 0 \\ -25 & 0 & 0 & 0 & 112.5 & -50 & 0 & 0 & -37.5 & 0 \\ -75 & 0 & 0 & -50 & -50 & 350 & 0 & -50 & -50 & 0 \\ 0 & 0 & 0 & 0 & 0 & 0 & 1 & 0 & 0 & 0 \\ 0 & 0 & 0 & 0 & 0 & 0 & 0 & 1 & 0 & 0 \\ 0 & 0 & 0 & 0 & -37.5 & -50 & 0 & 0 & 112.5 & 0 \\ 0 & 0 & 0 & 0 & 0 & 0 & 0 & 0 & 0 & 1\end{array}\right]$

While the vector of free terms will be: $4.730,2502.5,100,500,22500,4.230,32500,100,200,7502,300$. After the system solution by the method of Gauss the loads in nodes results as follows. $\Phi_{1}=210.825 \mathrm{~m}, \Phi_{2}=168.554 \mathrm{~m}, \Phi_{3}=100 \mathrm{~m}$, $\Phi_{4}=171.827 \mathrm{~m}, \Phi_{5}=231.687 \mathrm{~m}, \Phi_{6}=230.898 \mathrm{~m}, \Phi_{7}=100 \mathrm{~m}, \Phi_{8}=200 \mathrm{~m}, \Phi_{9}=246.535 \mathrm{~m}, \Phi_{10}=300 \mathrm{~m}$

The program in the programming language $\mathrm{C}+$, calculations of the filtration.

\#include <math.h>

\#include <stdio.h>

\#include <stdlib.h>

\#include <string.h>

\#define nelem 10

\#define $\mathrm{N} 10$

\#include "uti.h"

// functions

void write_matrix(char *name, int $m$, int $n$, float ${ }^{* *}$ mat);

void Donul_square_matrix(int $n$, float ${ }^{*}$ mat);

void transform _matrix(int $n$, float ${ }^{*} \mathrm{~F}$, float ${ }^{* *} \mathrm{~A}$, float ${ }^{*} \mathrm{~B}$,int ${ }^{*} \mathrm{KF}$ );

int the procedure _ gauss(float ${ }^{\star *} a$, float $^{*} x$, float $\left.{ }^{\star} b\right)$;

void write_vector(char *name, int $n$, float ${ }^{*}$ vec);

void Multiply_matrix_with_constant(int $\mathrm{n}$, float ${ }^{* *} \mathrm{~A}$, float cste);

/I -

int main(int argc, char *argv[])

$\left\{\right.$ int i,j,k,i1,i2,i3,ig,jg, ${ }^{* *}$ elems, ${ }^{*} \mathrm{KF}$;

float ${ }^{*} \mathrm{x},{ }^{*} \mathrm{y},{ }^{*} \mathrm{~F},{ }^{* *} \mathrm{kel},{ }^{*} \mathrm{~B},{ }^{* *} \mathrm{kglobal}$;

float x1, y1, x2, y2, x3, y3, a1, a2, a3, b1, b2, b3, c1,c2,c3,DET,T,konstante;

kel=Allocate_matrix $(0,2,0,2)$;

elems = Allocate_matrix_int $(0,9,0,2)$;

$x=$ Allocate_vector $(0,9)$;

$y=$ Allocate_vector $(0,9)$;

$\mathrm{F}=$ Allocate_vector $(0,9)$;

$\mathrm{KF}=$ Allocate_vector_int $(0,9)$;

B=Allocate_vector $(0,9)$;

kglobal=Allocate_matrix $(0,9,0,9)$;

Donul_square_matrix $(9, \mathrm{kglobal})$;

elems $[0][0]=1 ; \operatorname{elems}[0][1]=5 ;$ elems $[0][2]=6$;

elems[1][0] = 5; elems[1][1] = 9; elems[1][2] = 6 ;

elems[2][0] = 6; elems[2][1] = 9; elems[2][2] = 10;

elems[3][0] = 2; elems[3][1] = 1 ; elems[3][2] = 4;

elems[4][0] = 1; elems[4][1] = 6; elems[4][2] = 4; 
elems[5][0] $=4 ; \quad$ elems[5][1] = 6; $\quad$ elems $[5][2]=8 ;$ elems $[6][0]=6 ; \quad$ elems $[6][1]=10 ;$ elems $[6][2]=8 ;$ elems[7][0] = 3; $\quad$ elems[7][1] $=2 ; \quad$ elems[7][2] $=4$; elems $[8][0]=4 ; \quad$ elems[8][1] $=7 ; \quad$ elems[8][2] $=3$; elems $[9][0]=4 ; \quad$ elems[9][1] $=8 ; \quad$ elems $[9][2]=7$; $x[0]=1 . ; \quad y[0]=3.0 ;$ $x[1]=1 ; y[1]=5.0 ;$ $x[2]=1.0 ; y[2]=7.0$; $x[3]=2.0 ; y[3]=5.0$; $x[4]=2.0 ; y[4]=1.0$;

$x[5]=3.0 ; y[5]=3.0$;

$x[6]=3.0 ; y[6]=7.0$;

$x[7]=4.0 ; y[7]=5.0$;

$x[8]=4.0 ; y[8]=1.0$;

$x[9]=5.0 ; y[9]=3.0$;

$\mathrm{T}=100$.

$F[2]=100.0 ; F[6]=100.0 ; F[7]=200 . ; F[9]=300.0$;

$\mathrm{B}[0]=4.73 ; \mathrm{B}[1]=2.5 ; \mathrm{B}[2]=0.0 ; \mathrm{B}[3]=0.0 ; \mathrm{B}[4]=4.23 ; \mathrm{B}[5]=0.0$;

$B[6]=0 . ; B[7]=0 . ; B[8]=2.0 ; B[9]=0.0$;

$\mathrm{KF}[0]=1 ; \mathrm{KF}[1]=1 ; \mathrm{KF}[2]=0 ; \mathrm{KF}[3]=1 ; \mathrm{KF}[4]=1 ; \mathrm{KF}[5]=1$;

$K F[6]=0 ; K F[7]=0 ; K F[8]=1 ; K F[9]=0$;

for $(\mathrm{k}=0 ; \mathrm{k}<$ nelem; $\mathrm{k}++)$

$\{\mathrm{i} 1=$ elems[k][0]; $\mathrm{x} 1=x[11-1] ; \mathrm{y} 1=y[11-1] ;$

i2 = elems[k][1]; $x 2=x[i 2-1] ; y 2=y[i 2-1] ;$

i3 = elems[k][2]; $x 3=x[i 3-1] ; y 3=y[i 3-1]$;

$\mathrm{DET}=\mathrm{x} 2^{*} \mathrm{y} 3+\mathrm{x} 1^{*} \mathrm{y} 2+\mathrm{x} 3^{*} \mathrm{y} 1-\mathrm{x} 2^{*} \mathrm{y} 1-\mathrm{x} 3^{*} \mathrm{y} 2-\mathrm{x} 1^{*} \mathrm{y} 3$;

printf ("InDET=\%.1fln",DET);

printf ("In");

system("PAUSE");

$a 1=x 2^{*} y 3-x 3^{*} y 2 ; a 2=x 3^{*} y 1-x 1^{*} y 3 ; a 3=x 1^{*} y 2-x 2^{*} y 1$;

b1 $=y 2-y 3 ; b 2=y 3-y 1 ; b 3=y 1-y 2 ;$

$c 1=x 3-x 2 ; c 2=x 1-x 3 ; c 3=x 2-x 1$;

Constant $=\mathrm{T} /\left(2^{*} \mathrm{DET}\right)$;

printf ("In a1=\%.1f; a2=\%.1f; a3=\%.1f;'ln",a1,a2,a3);

printf ("In b1=\%.1f; b2=\%.1f; b3=\%.1f;'ln",b1,b2,b3);

printf ("In c1=\%.1f; c2=\%.1f; c3=\%.1f;'In",a1,a2,a3);

system("PAUSE");

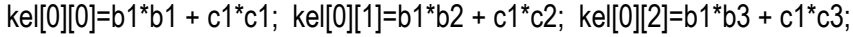

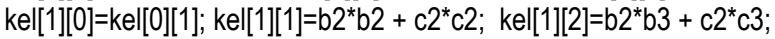

kel[2][0]=kel[0][2]; kel[2][1]=kel[1][2]; kel[2][2]=b3*b3 $+c 3^{*} c 3$;

Multiply_matrix_with_constant( 3 , kel, constant);

write_matrix("Elementary Matrix ", 3, 3, kel );

system("PAUSE");

for $(i=0 ; i<3 ; i++)$

$\{$ for $(j=0 ; j<3 ; j++)$

$\{$ switch(i)\{ case 0 :

ig $=\mathrm{i} 1-1$; break; case 1 :

ig $=$ i2-1; break; case 2 :

ig $=$ i3-1; break;

\} switch(j) \{

case 0 :

$\mathrm{jg}=\mathrm{i} 1-1$; break; case 1 :

jg $=$ i2-1; break; case 2: 


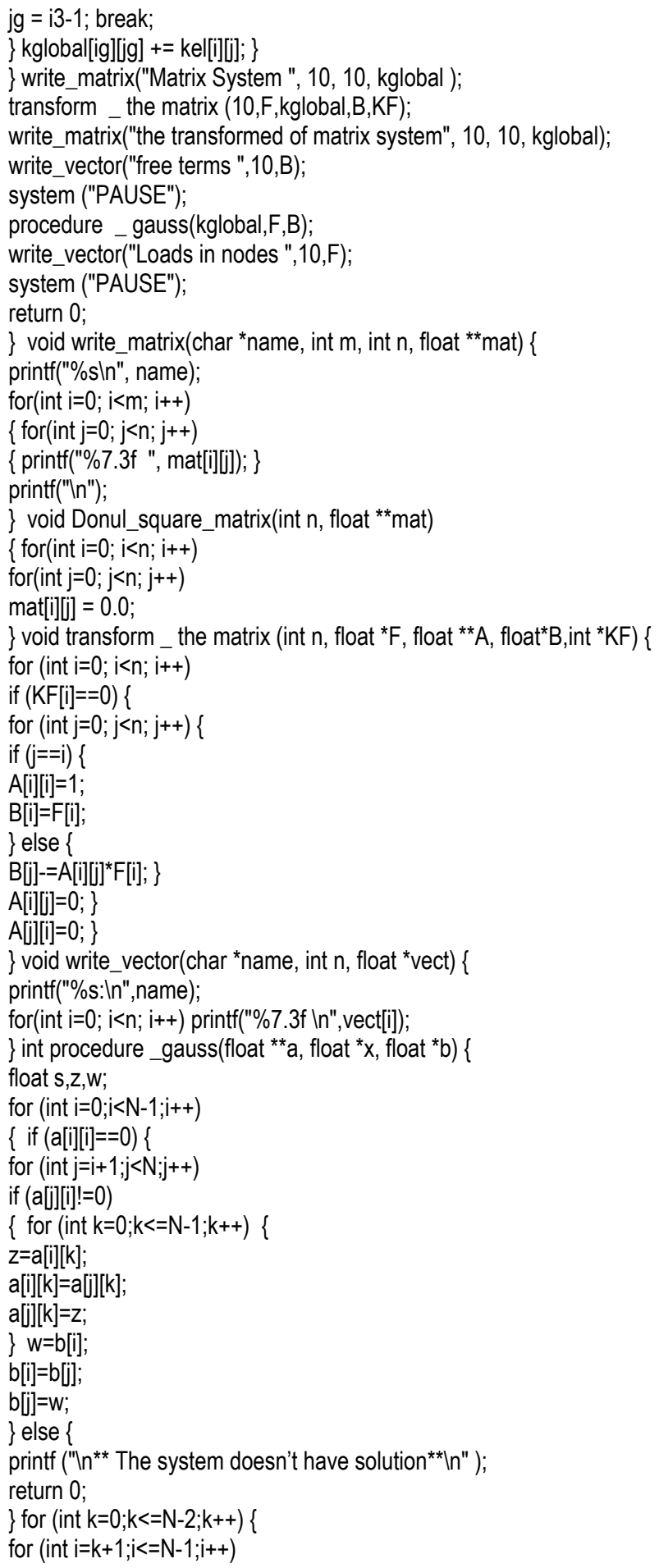




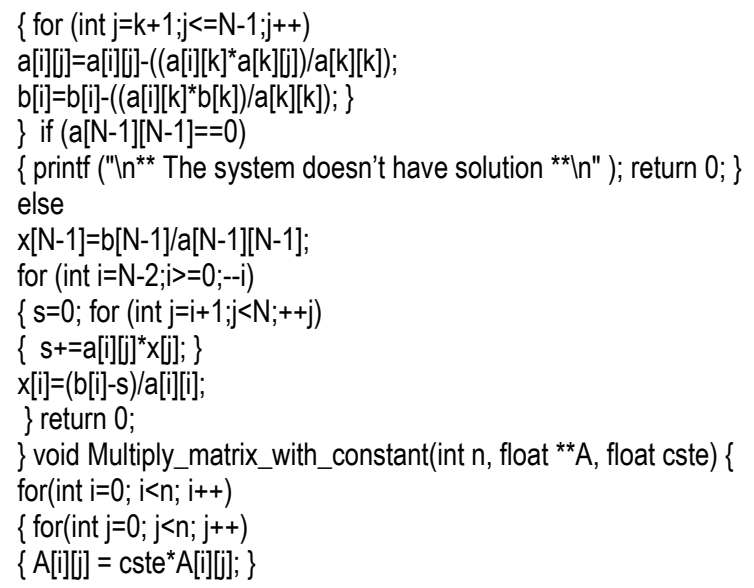

\section{Conclusion}

This scientific paper is a paper that treats the problem of water filtration with the finite elements method in the plan of DIRIHLE-NEUMANN.

Problems are determined by numerical methods with finite elements in which concrete results are obtained of water filtration, which are solved by matrix equations. Besides matrix calculations is also designed the calculation program in the programming language $\mathrm{C}+$.

Finite elements method applies a wide implementation not just in aquifer areas but other current water problems. These used equations in this scientific paper are based in two conditions of appearance: DIRIHLE type and NEUMANN type.

All the results of both conditions are entered into the software program $C+$ which program has determined the direction of the quantities of water filtrations in the Sibovc study area.

During the exited analyses results and obtained values from the DIRIHLE and NEUMANN model and calculating $C$ + program are given these findings:

Area towards to $Y$-axis $Y$ in nodes 1,2,3 and 4 and 1,4,6 and 5 are current areas with greater amounts of water $q=$ $2.5 \mathrm{~m} 3 /$ day, which means that water filtration in these nodes is with large quantities.

Area in points of nodes 5, 9 and 6 are areas with little impact on the aforementioned location that water filtration is not sufficient quantity and less elastic.

Area in points of nodes 9,6 and 10 water filtration affects less in this area and we based program $C+$ equation has no solution.

Area in points of nodes 5, 6 and 9 shows an internal filtration flux and density volume and water distributed in equally way in knots.

Area in points of nodes 5, 9 and 3 represents the greatest flux of flow water due to flow of water in this triangle.

By this program can be proved that each of the terms of the Drihile equation vector and Neumann type will provide adequate choice of quantity of water which is filtered in equal order of area which is analysed. Can emphasize that enables $\mathrm{C}+$ program enables orientation respectively waterfront area mapped to filter larger quantities of water with triangular outline.

\section{References}

Skender Osmani "Numerical Methods" Scientific Library, University of Tirana Polytechnic number 519.612 / 0737 Tirana, Albanian 1997. Chang, H.; Henriquez, A.; Honey, M.; Light, D.; Moeller, B.; Ross, N. (1998), The Union City story. Educational Development Centre. Mchung, J. A. (1990) Algorithmic Graph Theory, Prentice Hall.

Object-oriented, programming in $C^{+}$for $C$ and Java programmers February 2010, Department of Computer Science, Alborg University, Denmark.

Agni Dika "Object Oriented Programming in C + +" European University Southeast Faculty of Sciences and Technologies, CIP - Library cataloguing in publication National University "Sv. Kliment Ohridski ", 2008, Skopje. Macedonia. 
Frank L. Friedman, Elliot B. Koffman Problem Solving, Abstraction, and Design Using C++ Pearson Addison Wesley, USA 2004. D. S. Malik C++ Programming: From Problem Analysis to Program Design Course Technology, Massachusetts USA 2002. Agni Dika H. Algorithms, basic knowledge, with programs in C + + Faculty of Electro, Pristine, 2002, 2004. Republic of Kosovo. Frank L. Friedman, Elliot B. Koffman Problem Solving, Abstraction, and Design Using C++ Pearson Addison Wesley, USA 2004. Stanley B. Lippman, Josée Lajoie, Barbara E. Moo C++ Primer, Fourth Edition Addison-Wesley, USA 2005.

Organizata Botërore e Shëndetsisë,basic preconditions 2010.

Geography study, World water resourses, 2008.

Politika e Qeverisë për Ujëra,Republika e Kosoves, 2012. 